\title{
El dolor muscular referido es primariamente de origen periférico: la teoría de "barrera-presa"
}

\author{
A. Farasyn ${ }^{1}$ y A. Cuesta-Vargas ${ }^{2}$ \\ ${ }^{1}$ Departamento de Fisioterapia. Universidad Libre de Bruselas. Bélgica. ${ }^{2}$ Departamento de Psiquiatría y \\ Fisioterapia. Universidad de Málaga. Málaga
}

Farasyn A, Cuesta-Vargas A. El dolor muscular referido es primariamente de origen periférico: la teoría de "barrera-presa”. Rev Soc Esp Dolor 2013; 20(6): 301-307.

\begin{abstract}
The mechanisms leading to the frequent occurrence of referred muscle pain (RP) are largely unknown. It is currently postulated that a chain reaction is activated implicating triggering of an ever-increasing number of afferent neurons and facilitation of the synaptic connection at the level of the spinal cord, causing more distally a distributed RP via convergent efferent pathways. The human interpretation of this phenomenon can be misleading when the tested person positions, after a few seconds, his RP distally from the site of pressure to the distally located RP area. As a result of what we have found in our experimentally induced RP probes, we hypothesize that deep pressure on a myofibrosis will, in the first place, within a few seconds, increase the nociceptive excitability of the afferent sensitive nerves from the RP area to the local spot of pressure on the muscle, which in return will, in the second place, excite the whole dependent RP area. The mechanism for referred muscle pain proposed in this article is linked to the "prelocal hyper-excitability theory" or "barrier-dam theory". The afferent sensitive peripheral nerves might be entrapped in local muscle hardenings (barrier-dam) with the consequence of the hyper-excitation of pre-local nerves between the distally referred pain area and the local muscular zone of tenderness. The primary pathogenesis of referred muscle pain is likely to be a peripheral sensitization with additionally a central modulation and not vice versa. Clinical and fundamental studies with experimentally induced RP are nevertheless needed to examine the hypothesis.
\end{abstract}

Key words: Myofascial pain. Neuropatic pain. Referred pain.

Recibido: 15-05-12.

Aceptado: 21-08-12

\section{RESUMEN}

Los mecanismos que llevan a una alta incidencia del dolor muscular referido (DMR) son en gran medida desconocidos. En la actualidad, se postula que se produce la activación de una reacción en cadena que implica el desencadenamiento de la excitación de neuronas aferentes y la facilitación de las conexiones sinápticas al nivel de la médula espinal, lo cual produce el DMR distribuido más distalmente a través de las vías convergentes eferentes. Como resultado de los hallazgos de nuestros estudios del DMR inducido experimentalmente, se puede plantear la hipótesis de que la presión profunda sobre la zona de miofibrosis incrementará primariamente la excitabilidad nociceptiva de los nervios aferentes sensitivos desde el área de DMR hasta el sitio de la presión local en el músculo en pocos segundos, lo cual a su vez excitará secundariamente el área total dependiente de DMR. El mecanismo propuesto en este artículo para el dolor muscular referido está ligado a la teoría de la "hiperexcitabilidad pre-local" o la "teoría de barrera-presa". Los nervios aferentes sensitivos periféricos pueden ser atrapados en los endurecimientos musculares locales (barrera-represa), con la consecuencia de la hiperexcitación de los nervios pre-locales entre el área distal de dolor referido y la zona local muscular de sensibilidad dolorosa a la presión. La patogénesis primaria del dolor muscular referido puede ser probablemente la sensibilización periférica con la modulación central adicional y no viceversa. Sin embargo, para verificar esta hipótesis, son necesarios estudios fundamentales clínicos con el DMR inducido experimentalmente.

Palabras clave: Dolor miofascial. Dolor neuropático. Dolor referido.

\section{INTRODUCCIÓN}

El término dolor muscular referido (DMR) ha sido usado para describir el dolor urente percibido en el sitio 
adyacente al área local de sensibilidad muscular dolorosa (1-4). Es comúnmente aceptada la teoría de la proyección convergente de Ruch (5), modificada por Mense (6), sobre la sensibilización de las vías centrales nociceptivas, aunque esta no provee una explicación de los mecanismos musculares primarios (7).

El dolor referido que resulta de las fuentes de dolor muscular es comúnmente acompañado por la hiperalgesia secundaria y cambios tróficos $(8,9)$. Los mecanismos patofisiológicos responsables del DMR no han sido clarificados completamente, y la razón por la cual el DMR es más prevalente en unos sujetos y no en otros es desconocida. La teoría de la sensibilización central explica el DMR como el resultado de la aparición de dos nuevos campos receptivos e indica que los estímulos nóxicos provenientes del tejido muscular pueden producir la abertura de fibras aferentes convergentes latentes en el asta dorsal de la médula espinal $(10,11)$. La sensibilización central tiene importancia clínica debido a que a través de ella se puede explicar la hiperalgesia y la propagación del dolor en los pacientes. Puesto que el estímulo nóxico que actúa sobre el músculo con la densidad incrementada de nociceptores provocaría más dolor, el incremento en la densidad de nociceptores constituye el mecanismo periférico de hiperalgesia (6-12).

El resultante incremento de respuestas sinápticas incrementa el establecimiento de conexiones sinápticas excitatorias en las vías aferentes nociceptivas y contribuye de manera significativa a la hipersensibilidad al dolor (13). Los estudios de neuroimagen de dolor en humanos y animales con tomografía por emisión de positrones (PET) y resonancia magnética funcional (fMRI) han encontrado incrementos somatotópicamente organizados de la excitación en el asta dorsal de la médula espinal e incrementos similares en varias áreas del cerebro relacionadas con el dolor (14). Estos estudios han confirmado el indicio de que la sensibilización central está involucrada en la generación del DMR.

Otra teoría (9) explica que el DMR es generado por las estructuras somáticas profundas (p.ej. otros músculos), y por lo tanto no se explica por los mecanismos de la sensibilización central de neuronas convergentes en su forma original, puesto que existe poca convergencia desde los tejidos profundos en las neuronas del asta dorsal. Esta teoría está basada en la suposición de que la abertura de estas conexiones se produce por la aferentación nociceptiva desde los músculos esqueléticos y de que el dolor referido a los miotomas fuera de la lesión ocurre por la propagación de la sensibilización central a los segmentos medulares adyacentes. Sin embargo, los estudios del DMR inducido experimentalmente por Laursen y colaboradores (15-19) han mostrado que la producción del dolor muscular referido es dependiente del dolor muscular local y muestra una correlación con este.

La hipótesis de los puntos gatillo miofasciales (PGM) de Travell y Simons (3) sugiere que las neuronas nocicep- tivas del asta dorsal que reciben estímulos convergentes de varios tejidos confunden a los centros cerebrales superiores con respeto a la identificación de la fuente de entrada. La presión mecánica (Fig. 1) sobre el punto gatillo incrementa la actividad neural (A y B) así como las sensaciones referidas (dolor, parestesia) al área objetivo (C y D) con la retroalimentación a la médula espinal para incrementar la carga de solicitación de fondo. Otros estímulos llegan a la médula espinal de los puntos gatillo distales y áreas disfuncionales adicionales (E y F). Los hallazgos de que los PGM causan el DR ponen en seria duda la validez interna del concepto (19). En casos crónicos el DMR se explica por el ciclo de retroalimentación mantenido por los centros cerebrales superiores que reciben impulsos originados en los PGM. Cuando la presión digital profunda se aplica sobre el músculo normal o en un sujeto que presenta la lesión fibrótica lisa (miofibrositis) en el músculo, el DMR puede surgir como un área de hiperalgesia cutánea, adyacente a la zona dolorosa a la presión, o completamente alejada de esta.

En la actualidad, se postula que se produce la activación de una reacción en cadena que implica el desencadenamiento de la excitación de neuronas aferentes y la facilitación de las conexiones sinápticas al nivel de la médula espinal, lo cual produce el DMR distribuido más distalmente a través de las vías convergentes eferentes. La interpretación humana de este fenómeno puede ser errónea, después de pocos segundos, cuando la persona examinada ubica su DMR en una zona distal al sitio de la presión.

\section{DESARROLLO}

\section{Nueva hipótesis}

\section{Miofibrosis lisa (PGM latentes)}

Como resultado de los hallazgos de nuestros estudios del DMR inducido experimentalmente, la hipótesis de los puntos gatillo miofasciales (PGM) de Farasyn (20) sugiere que: la presión profunda sobre la zona de miofibrosis incrementará, en pocos segundos, la excitabilidad nociceptiva de los nervios aferentes sensitivos desde el área de DMR hasta el sitio de la presión local en el músculo, lo cual a su vez excitará el área total dependiente de DMR (Fig. 2).

\section{Miofibrosis dura (PGM activos)}

En el caso de los músculos que contienen la miofibrosis dura (PGM activos), la parte pre-local del nervio aferente periférico es permanentemente perturbada, "corriente arriba" desde el área de DMR hasta la zona de sensibilidad dolorosa a la palpación, como resultado de los impulsos aferentes facilitados de manera permanente al nivel de los 


\section{(1) Presión mecánica}

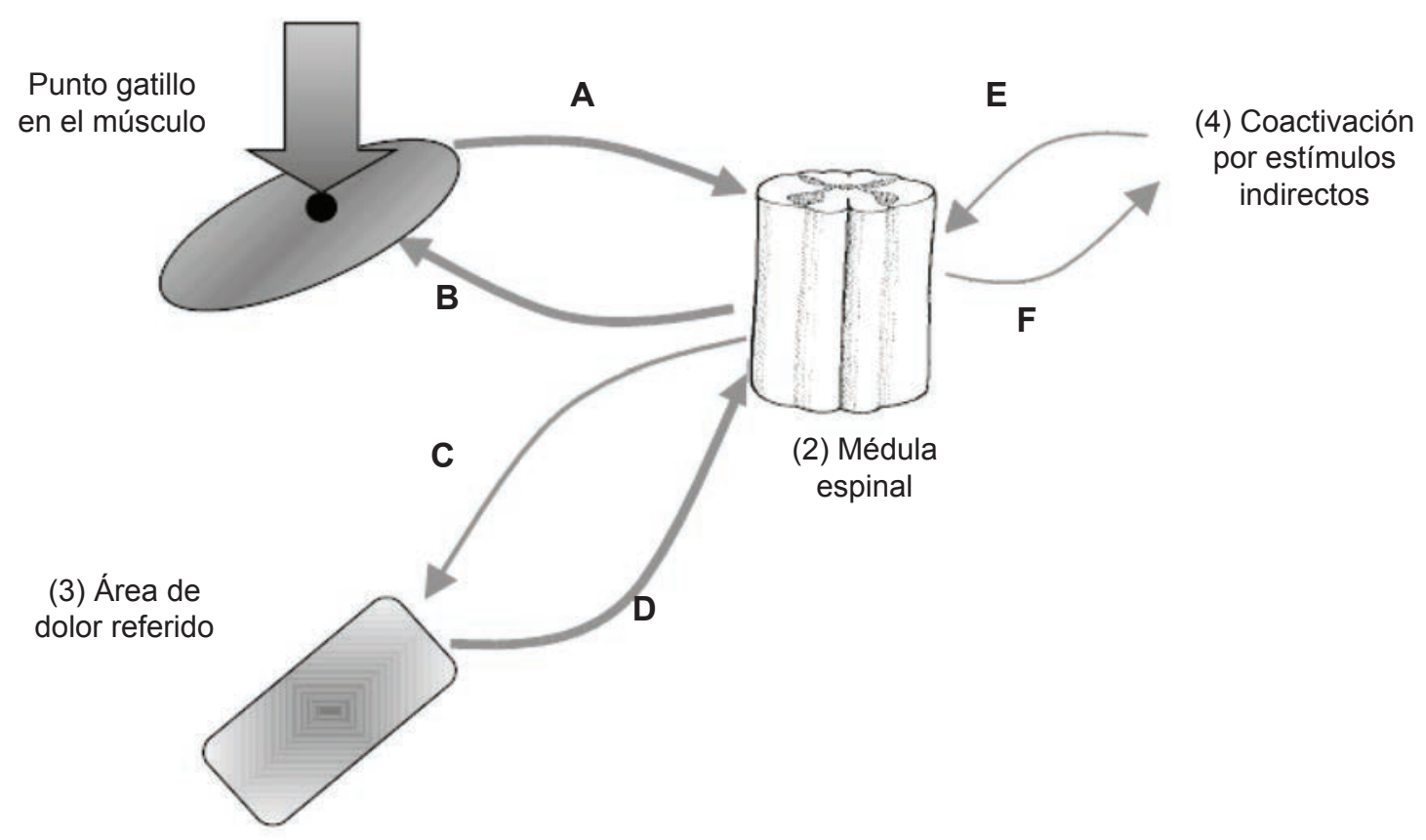

Fig. 1. Hipótesis de los puntos gatillo miofasciales (PGM) de Travell y Simons (1998): la presión mecánica sobre el punto gatillo incrementa la actividad neural (A y B) así como las sensaciones referidas (dolor, parestesia) al área objetivo (C y D) con la retroalimentación a la médula espinal para incrementar la carga de solicitación de fondo. Otros estímulos llegan a la médula espinal de los puntos gatillo distales y áreas disfuncionales adicionales (E y F).

nervios periféricos post-locales, "corriente abajo" desde la zona de atrapamiento y hasta la médula espinal. La modulación al nivel de la médula hace que la "puerta" permanezca permanentemente "abierta" hasta el tálamo para el ulterior procesamiento de los patrones de DMR “crónicamente presentes".

El dolor fantasma puede aparecer cuando la presión mecánica se usa para provocar el dolor muscular referido en el lado del miembro inferior amputado, en el sentido de que en el área de DMR como tal este es reconocido como un patrón de dolor que fue "aprendido" antes, en el periodo previo a la amputación. Es obvio que en este caso el área de DMR no está presente corporalmente sino que su patrón es reconocido "como tal" a nivel de los centros cerebrales superiores.

La coactivación de otros nervios aferentes sensitivos pre-locales que se originan de los músculos paralelos sanos (Fig. 2D), los cuales están adicionalmente semiactivados, hace que el sujeto se da cuenta en mayor grado del DMR y/o de las áreas adyacentes de DMR (Fig. 2E). Cuando provocamos experimentalmente un dolor referido a través de la presión mecánica profunda sobre el músculo con la miofibrosis dura, esto incrementará, en primer lugar, la entrada de las señales “corriente arriba”, desde el área de DMR hasta el sitio de la presión local en el músculo, y el área total de DMR se volverá hiperactivada al máximo. Este fenómeno resulta, en segundo lugar, en la facilitación incrementada del componente de nervios aferentes sensitivos poslocales, los cuales a su vez trasmiten las señales a los centros cerebrales superiores donde el dolor es experimentado como una sensación "intolerable" de DMR. La retroacción simpática incrementada, en calidad de un reflejo patofisiológico a nivel de los segmentos dependientes de la médula espinal, produce más reacciones locales (vasoconstricción cutánea, sudoración, etc.).

La terapia de los "puntos gatillo" que refiere al DMR es probablemente incorrecta, porque la existencia de una relación directa entre las vías neurológicas eferentes y aferentes como la explican Travell y Simons (3) nunca fue probada adecuadamente con sus respectivos sustratos anatómicos. La activación de llamadas zonas de PGM satélites no es modulada a nivel de la médula espinal, sino que tiene su origen en las interacciones de los nervios periféricos aferentes paralelos. La importancia de la médula espinal $\mathrm{y}$, especialmente, de la teoría de la sensibilización central puede haber sido sobreestimada. No es el cerebro humano 


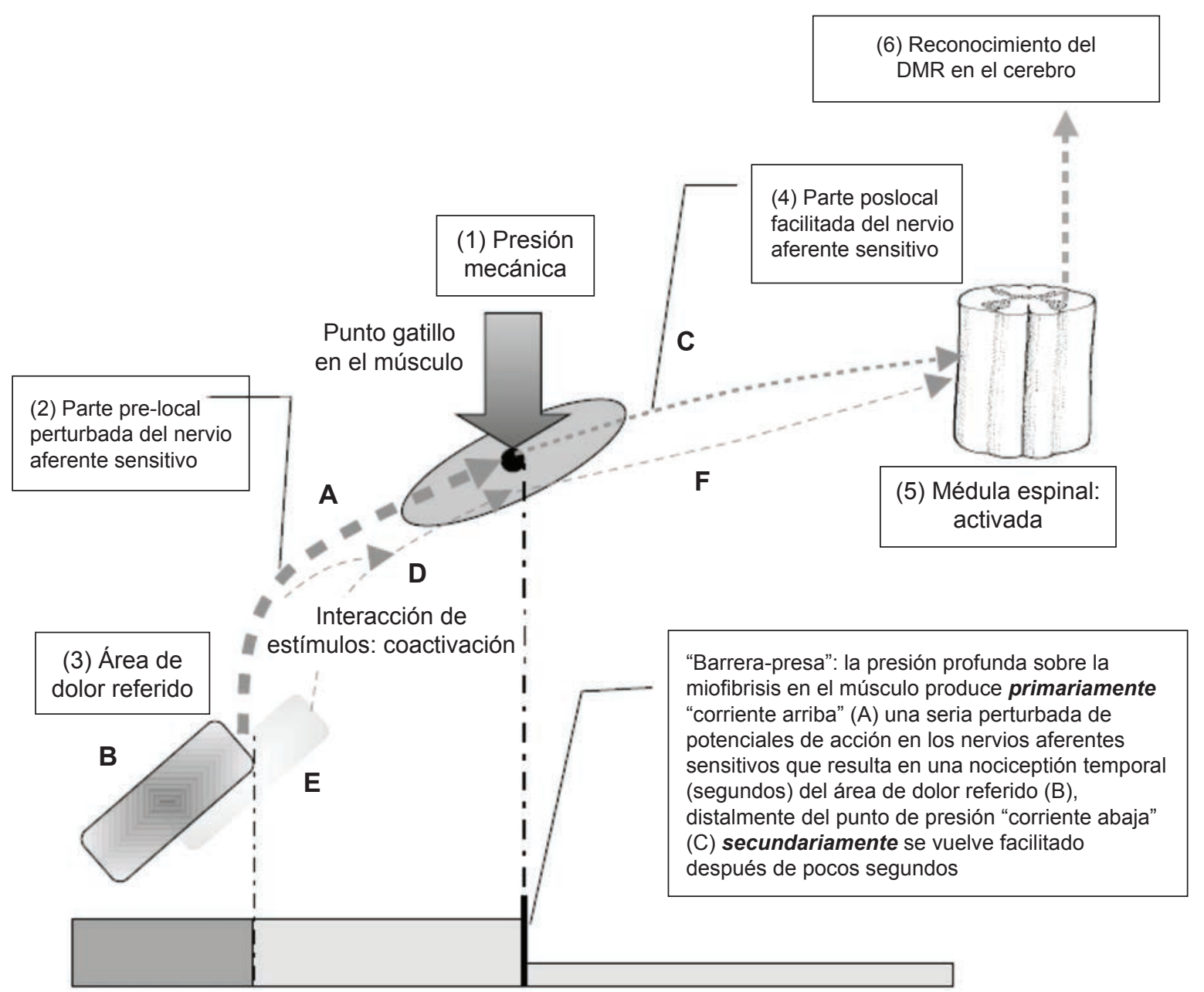

Fig. 2. Nueva hipótesis del dolor referido: la "teoría de barrera-presa" (Farasyn, 2007). La presión mecánica sobre el punto gatillo incrementa la actividad neural primariamente en la parte pre-local (A) así como en el área dependiente de dolor referido (dolor, parestesia) (B), y secundariamente facilita después de pocos segundos el nervio aferente periférico sensitivo poslocal (C) la transmisión a los centros superiores del cerebro donde el área de dolor referido es finalmente reconocida como tal. De hecho, cualquier dolor referido presente en la superficie corporal puede ser explicado como una neuropatía por atrapamiento de un nervio periférico sensitivo. Otros estímulos interactuan a través de la red de nervios sensitivos (D) de modo que la coactivación ocurre en las áreas adyacentes de dolor referido (E) y facilita el resto de nervios aferentes (F).

que malinterpreta el fenómeno de PGM. La llamada teoría de la proyección convergente, al parecer, ha sido aceptada por la comunidad científica en ausencia de la evidencia definitiva de su validez.

\section{Breve reseña histórica}

La movilización miofascial por fricción transversa profunda es comúnmente usada por los terapeutas manuales en los países del Extremo Oriente como Japón (shiatsu), Tailandia y la India, y es distinta del masaje tradicional occidental (sueco) $(21,22)$. Su aplicación tiene como objetivo la liberación de los patrones crónicos de tensión muscular a través del uso de la presión o fricción directa, principalmente sobre las partes centrales de los músculos.

Varias técnicas de tratamiento han sido sugeridas para el tratamiento de los endurecimientos musculares dolorosos y los síntomas referidos producidos por ellos. Estas técnicas incluyen, aparte de los tratamientos usuales hasta ahora en la fisioterapia como las aplicaciones de calor local y los estiramientos musculares, los tratamientos locales mecánicos especiales, como la aplicación de la "yuan zhen" ("aguja de punta redonda") $(23,24)$.

En Japón esta herramienta terapéutica es conocida como “enshin” y está siendo usada frecuentemente hoy en Japón 
para aplicar masaje sobre la espalda (33). Desde el punto de vista de la medicina convencional occidental, se supone que el uso de la movilización por fricción transversa profunda, empezando por la presión de $3-4 \mathrm{~kg} / \mathrm{cm}^{2}$, disminuye adherencias y dilacera los tejidos cicatrizales. Se presume que los endurecimientos musculares son el resultado residual de la respuesta inflamatoria incompleta antes de que el proceso de curación llegue a la fase de reparación y/o de regeneración (25-27).

En los años 1980 terapeutas japoneses (28-29) modificaron la herramienta tradicional "enshin" añadiéndole el mango para mejor agarre y transformándola en una barra $\mathrm{T}$ miofascial de bronce $(0,80 \mathrm{~kg})$ usada para aplicar la técnica terapéutica específica de masaje por fricción transversa profunda. Esta técnica es caracterizada por el incremento de la presión empezando de $5-10 \mathrm{~kg} / \mathrm{cm}^{2}$, aplicada a las capas profundas del músculo durante 30 minutos, una vez a la semana por dos semanas. Los efectos sobre las estructuras músculo-esqueléticas que se procura obtener con esta técnica son la regeneración de los tejidos conectivos cicatrizales y la reducción de los endurecimientos musculares como un posible mecanismo de alivio del dolor $(27,28)$. Nosotros hemos llamado a esta nueva técnica "roptroterapia" (29-31). La palabra roptroterapia es la combinación de "roptron", que significa "el nudo" en el griego clásico, y "terapia".

\section{Estudios clínicos}

Los resultados de diferentes ensayos clínicos aleatorios sobre el efecto de la roptroterapia aplicada sobre diferentes localizaciones anatómicas demuestran que los pacientes con el dolor muscular referido tratados con dos sesiones de roptroterapia ya no presentan el DMR clínicamente durante la tercera semana (Fig. 3). Cuando es aplicada la movilización por fricción transversa profunda al tejido fibrosítico (cicatrizal) en los endurecimientos musculares durante al menos dos sesiones semanales, probablemente se liberaron los atrapamientos de los nervios sensitivos, lo cual probablemente tuvo como resultado el reestablecimiento de la conducción normal de señales en los nervios aferentes sensitivos (Fig. 3) evidenciada en la evaluación durante la sesión de seguimiento de la tercera semana (20-31).

Estos hallazgos clínicos se evidenciaron principalmente en síndromes músculo-esqueléticos, como:

- Lumbalgia no específica con el DMR a la región posteroexterna de la cadera: también conocida como "pseudociatalgia", causada por el atrapamiento del nervio clúneo superior interno a nivel de los endurecimientos musculares en la región proximal del músculo glúteo medio y del tensor de la fascia lata.

- Cefalea tensional con el DMR a las regiones temporal, frontal y orbital del cráneo, causada por el atrapamiento del nervio auricular, el nervio transverso del cuello y los nervios occipitales a nivel de los endurecimientos musculares ubicados respectivamente en la parte media del músculo esternocleidomastoideo y en la parte superior del músculo trapecio.

- Lesiones por esfuerzos repetitivos del hombro con el DMR en el dorso del brazo y de la mano, causado por el atrapamiento de los ramos posteriores de nervios torácicos a nivel de los músculos romboides e infraespinoso.

La movilización por fricción transversa puede causar la remodelación del tejido cicatrizal presente en el músculo tratado, teniendo como resultado la liberación del atrapamiento, incluyendo la normalización de la excitación del nervio aferente sensitivo situado periféricamente (zona A y DMR zona B en la Figura 3). El nervio aferente sensitivo post-local (Fig. 3C), la transmisión a nivel del asta dorsal de la médula espinal y los centros superiores del cerebro pueden disminuir su nivel de excitación y después de pocas semanas llegar al estado clínico de total desaparecimiento de la zona del dolor referido. Se produce la apertura de la barrera-presa, y se normaliza la conducción de impulsos en el nervio periférico aferente (parte inferior de la figura 3). Los resultados sugieren que el fenómeno de DMR crónico no depende primariamente de la sensibilización central, sino más bien de la sensibilización periférica.

\section{CONCLUSIONES}

En el caso de los síndromes comunes de dolor muscular referido, la sensibilización medular o central no constituye un problema importante, pero la atención debe prestarse a lo que sucede "corriente arriba" del sitio de atrapamiento del nervio. Esta presunción puede llevar a una nueva hipótesis del dolor muscular referido: la "teoría de barrera-presa". Nuestros hallazgos clínicos contribuyen a la hipótesis de que cualquier dolor muscular referido tiene su origen primariamente en las estructuras musculares locales lesionadas, las cuales producen atrapamiento de los nervios sensitivos periféricos. Estos endurecimientos musculares pueden ser responsables de la conducción afectada en los nervios aferentes que atraviesan los músculos examinados, presentando el cuadro de dolor muscular referido. No parece ilógico suponer que el DMR a las zonas del cuerpo situadas distalmente asociado con los síndromes miofasciales de puntos gatillo es similar a, por ejemplo, el dolor urente en la meralgia parestésica (32), en la cual el nervio femorocutáneo sensitivo atrapado produce el DMR en la región anterior del muslo.

Nuestra teoría provee una explicación potencial para el mecanismo muscular primario en relación al dolor muscular referido o DMR. La suposición de que el dolor muscular referido se origina como parte del proceso de sensibilización central debe ser revisada a favor de la neuropatología de atrapamiento del nervio sensitivo periférico. Esta teoría 
(1) Movilización por fricción transversa profunda o "roptroterapia"
(2) Respuesta prelocal de fibras aferentes al estímulo disminuida

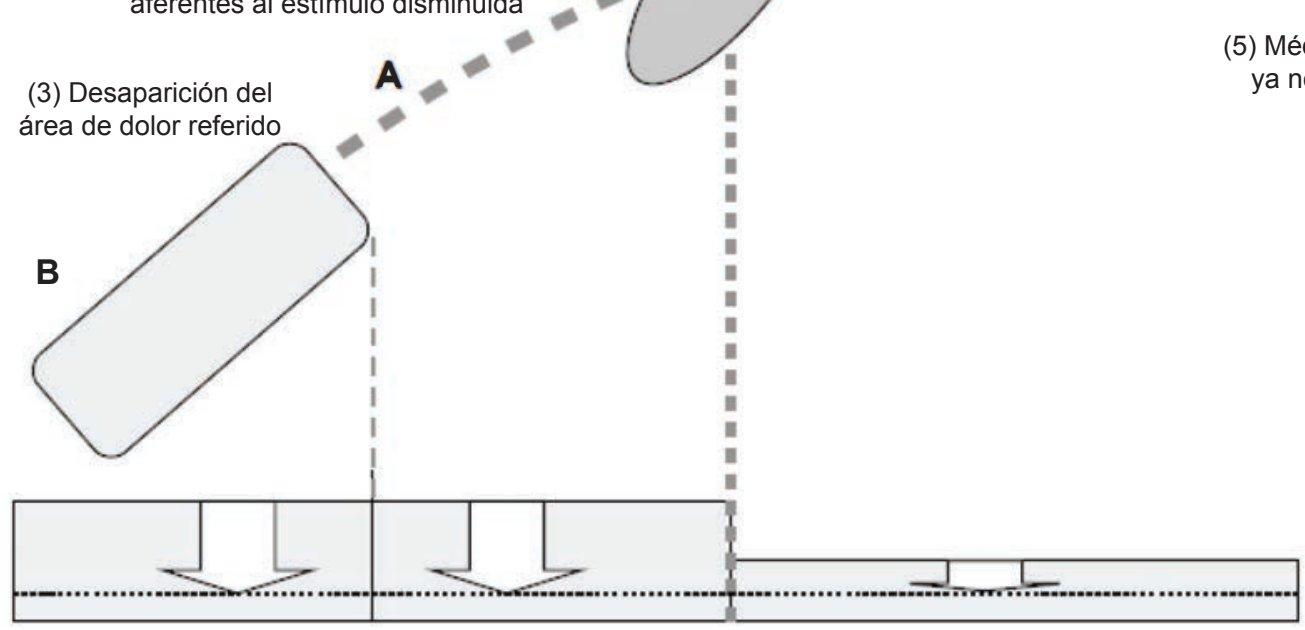

(6) No más percepción de dolor

(4) Respuesta poslocal de fibras aferentes al estímulo disminuida

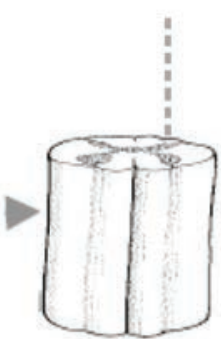

(5) Médula espinal: ya no activada

Fig. 3. Nueva hipótesis del dolor muscular referido: la "teoría de barrera-presa" (Farasyn, 2007). La movilización por fricción transversa profunda sobre el punto gatillo (miofibrosis presente en el músculo) o "roptroterapia" por 30 minutos sobre el PGM, $2 \mathrm{x}$ una sesión semanal, induce la remodelación de la actividad de los nervios aferentes sensitivos prelocalmente (A y DMR en el área B). Los nervios aferentes sensitivos poslocales (C), la transmisión de la señal en el asta dorsal de la médula espinal y los centros cerebrales superiores se encuentran menos activados durante los días y semanas siguientes.

de "barrera-presa" concuerda, de hecho, con la teoría de Quintner y Cohen (33), en la cual se postula que el dolor muscular referido es la hiperalgesia secundaria de origen neural periférico.

Por otra parte, la explicación occidental actualmente aceptada de sensibilización medular o central referente al DMR parece ser incorrecta, aunque incluye las estructuras anatómicas correctas como los nervios, la médula espinal y el sistema nervioso central. Otros estudios complementarios, como la medición a través de la electroneuromiografía de aguja para la calidad de neuroconducción en los nervios sensitivos y el estudio de los cambios histológicos en los tejidos cicatrizales producto del trabajo muscular excéntrico, serían sin embargo necesarios en el futuro.

\section{AGRADECIMIENTOS}

Estamos agradecidos al profesor Dr. P. Lievens, profesor Dr. R. Meeusen y al profesor Dr. Jo Nijs del Departa- mento de Fisiología Humana y Medicina Deportiva de la Facultad de Educación Física y Rehabilitación de la Vrije Universiteit Brussel por su apoyo en este estudio. También damos gracias al profesor Dr. P. Vaes por sus comentarios al manuscrito en inglés y a la Sra. Yvonne De Clercq por su invaluable ayuda.

CORRESPONDENCIA:

Antonio Cuesta-Vargas

e-mail: acuesta@uma.es

\section{BIBLIOGRAFÍA}

1. Kellgren J. Observations on referred pain arising from muscle. Clin Sci 1938;3:175-90.

2. Feinstein B, Langton J, Jameson R, Schiller F. Experiments on pain referred from deep somatic tissues. J Bone Surg 1954;36:981-97. 
3. Travell J, Simons D, editors. Myofascial pain and dysfunction. Baltimore: Lippincott Williams \& Wilkins; 1998.

4. Arendt-Nielsen L, Svensson P. Referred muscle pain: Basic and clinical findings. Clin J Pain 2001;17:11-9.

5. Ruch TC. Pathophysiology of pain. In: Ruch TC, Patton HD, eds. Physiology and biophysics. Philadelphia: Saunders; 1965. p. 345-63.

6. Mense S. Referral of muscle pain. Am Pain Society J 1994;3:1-9.

7. Boureau F, Delorme T, Doubrere J. Mechanisms of myofascial pain. Rev Neurol (Paris) 2000;156:4S10-4.

8. Vecchiet L, Vecchiet J, Giamberardino M. Referred muscle pain: Clinical and pathophysiologic aspects. Curr Rev Pain 1999;3:489-98.

9. Giamberardino M. Referred muscle pain/hyperalgesia and central sensitisation. J Rehabil Med 2003; 41(Supl.):85-8.

10. Travell J. Referred pain from skeletal muscle; the pectoralis major syndrome of breast pain and soreness and the sternomastoid syndrome of headache and dizziness. NY State J Med 1955;3:331-40.

11. Mense $\mathrm{S}$. Nociception from skeletal muscle in relation to clinical muscle pain. Pain 1993;54:241-89.

12. Simons D, Mense S. Diagnosis and therapy of myofascial trigger points. Schmerz 2003;6:419-24.

13. Salter M. Neurobiology of central sensitization. J Musculoskel 2002;10:23-43.

14. Manhofner C, Forster C, Birklein F, Neudorfer B, Hardwerker $\mathrm{H}$. Brain processing during mechanical hyperalgesia in complex regional pain syndrome: A functional MRI study. Pain 2005;114:93-103.

15. Laursen R, Graven-Nielsen T, Jensen T, et al. The effect of compression and regional anaesthetic block on referred pain intensity in humans. Pain 1999:80-257-63.

16. Laursen R, Graven-Nielsen T, Jensen T, Arendt-Nielsen L. The effect of differential and complete nerve block on experimental muscle pain in humans. Muscle Nerve 1999;22:156470.

17. Graven-Nielsen T, Arendt-Nielsen L, Svensson P, Jensen T. Quantification of local and referred pain in humans after sequential i.m. injections of hypertonic saline. Pain 1997;69:111-7.

18. Graven-Nielsen T, McArdle A, Phoenix J, et al. In vivo model of muscle pain: Quantification of intramuscular che- mical, electrical, and pressure change associated with saline-induced muscle pain in humans. Pain 1997;69:137-43.

19. Slater H, Arendt-Nielsen L, Wright A, Graven-Nielsen T. Sensory and motor effects of experimental muscle pain in patients with lateral epicondylalgia and controls with delayed onset muscle soreness. Pain 2005;14:118-30.

20. Farasyn A. Referred muscle pain is primarily peripheral in origin: The "Barrier-Dam" theory. Medical Hypotheses 2007;68:144-50.

21. Pearce J. Myofascial pain, fibromyalgia or fibrositis? Eur Neurol 2004;52:67-72.

22. Ernst E. Massage therapy for low back pain: a systematic review. J Pain Sympt Manag 1999;17:56-69.

23. Birch SJ, Ida J. Japanese acupuncture: A clinical guide. Brookline, Mass.: Paradigm Publ.; 1998.

24. Wang Z, Wang J. Ling Shu acupuncture. Anaheim, CA: Ling Shu Press; 2007.

25. Patino O, Novick C, Merlo A. Massage in hypertrophic scar. J Burn Care Rehabil 1999;20:268-71.

26. Cantu R, Grodin A. Myofascial manipulation: theory and clinical application. 2nd ed. Gaithersburg USA: Aspen Publ.; 2001.

27. Tsujii Y, ed. Myotherapy, treatment of muscle hardenings. Japan: Nagoya University College of Medical Technology Nagoya; 1993.

28. Nagata C, Tsujii Y. Myotherapy: A new approach to the treatment of muscle pain syndromes. J Manual Manipulat Ther 1997;5:87-90.

29. Farasyn A, Meeusen R. Effect of roptrotherapy on pressure pain thresholds in patients with non-specific low back pain. J Muscoskel Pain 2007; 15:41-53.

30. Farasyn A, Meeusen R, Nijs J. A pilot randomized placebo-controlled trial of roptrotherapy in patients with subacute low back pain. J Back Musculoskel Rehabil 2007,14:111-17.

31. Farasyn A. Release of myofascial pain with deep cross-friction named "roptrotherapy". Int J Ther Massage Bodyw 2010;3:36-37.

32. Russo L, Firestone M, Mandler R, Kelly Jr J. Nerve conduction studies of the lateral femoral cutaneous nerve. Implications in the diagnosis of meralgia paresthetica. Am J Electroneurodiagnostic Technol 2005;45:180-5.

33. Quintner J, Cohen M. Referred pain of peripheral nerve origin: An alternative to the "myofascial pain" construct. Clin J Pain 1994;10:243-51. 